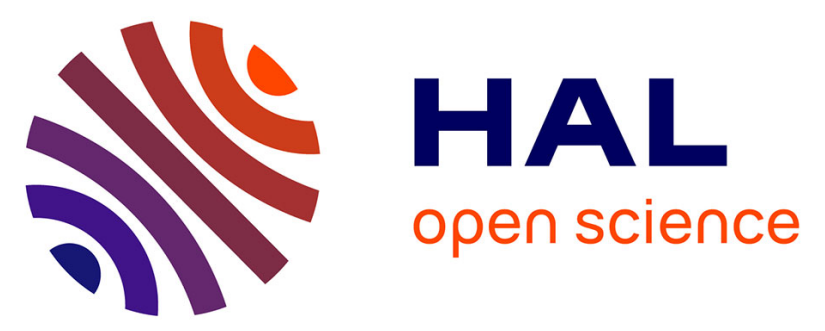

\title{
Cryostat pour l'étude d'échantillons irradiés à basse température par résonance paramagnétique électronique
}

Tilo Kester, Bernard Lamotte, Michel Brochier, Jacques Doulat

\section{To cite this version:}

Tilo Kester, Bernard Lamotte, Michel Brochier, Jacques Doulat. Cryostat pour l'étude d'échantillons irradiés à basse température par résonance paramagnétique électronique. Revue de Physique Appliquée, 1966, 1 (3), pp.249-251. 10.1051/rphysap:0196600103024900 . jpa-00242728

\section{HAL Id: jpa-00242728 https://hal.science/jpa-00242728}

Submitted on 1 Jan 1966

HAL is a multi-disciplinary open access archive for the deposit and dissemination of scientific research documents, whether they are published or not. The documents may come from teaching and research institutions in France or abroad, or from public or private research centers.
L'archive ouverte pluridisciplinaire HAL, est destinée au dépôt et à la diffusion de documents scientifiques de niveau recherche, publiés ou non, émanant des établissements d'enseignement et de recherche français ou étrangers, des laboratoires publics ou privés. 


\title{
CRYOSTAT POUR L'ÉTUDE D'ÉCHANTILLONS IRRADIÉS A BASSE TEMPÉRATURE PAR RÉSONANCE PARAMAGNÉTIQUE ELECTRONIQUE
}

\author{
Par Tilo KeSter ( ${ }^{\mathbf{1}}$ ), Bernard LAMOTte ( ${ }^{\mathbf{1}}$ ), \\ Laboratoire de Résonance Magnétique. \\ Michel BROCHIER, \\ Section des Ateliers de Mécanique, \\ et Jacques DOULAT, \\ Section de Physico-Chimie et Basses Températures, Centre d'Études Nucléaires de Grenoble, France.
}

\begin{abstract}
Résumé. - Une courte description technique d'un cryostat RPE est suivie de la discussion de ses performances : on décrit les procédés utilisés pour faire varier la température, transférer et recuire l'échantillon, ainsi que le dispositif d'irradiation U. V. in situ.
\end{abstract}

Abstract. - A short technical description of an ESR cryostat is followed by a discussion of its performance. The techniques which permit variable temperature operation, sample transfer and annealing, and u. v. irradiation of the sample " in situ" are described.

I. Introduction. - Nous décrivons un cryostat à hélium liquide pour la résonance paramagnétique électronique $(\lambda=3 \mathrm{~cm})$, qui permet le transfert d'échantillons irradiés à basse température sans réchauffement, et le changement rapide d'échantillon en cours d'expérience. Il présente en outre les possibilités suivantes :

Variation de la température entre 1,3 et 80 oK.

Irradiation ultraviolette " in situ ".

Recuit d'un échantillon au-dessus de $80^{\circ} \mathrm{K}$.

Étude à haute sensibilité (modulation du champ à $100 \mathrm{kHz}$ ) de l'anisotropie des spectres de RPE par rotation de l'échantillon dans le cryostat.

Pour répondre à ces exigences, le choix de la géométrie de la cavité (" autour ) de laquelle le cryostat est bâti) est déterminant. Nous avons opté pour une cavité cylindrique, excitée suivant le mode TE 011 par un tube coaxial, placé en position excentrique.

II. Description. - Le cryostat, placé dans l'entrefer d'un aimant VARIAN 9 pouces de $75 \mathrm{~mm}$ est construit en acier inoxydable.

Il est entièrement démontable, les parties froides étant assemblées par joints d'indium.

Il se compose de cinq parties distinctes :

le vase d'azote liquide,

le ballon d'hélium liquide (enceinte E1),

(1) Des éléments de ce travail appartiennent aux thèses de doctorat de MM. Bernard Lamotte (Faculté des Sciences de Grenoble, France) et Tilo Kester (Université de Giessen, Allemagne, D 26), boursier de l'OTAN puis de l'EURATOM. le tube central contenant l'expérience proprement dite (enceinte E2),

l'ensemble porte-échantillon,

l'unité d'irradiation U. V.

La figure 1 montre le cryostat assemblé. La partie centrale est représentée séparément sur la figure 2.

III. Fonctionnement et performances. - Le tableau ci-dessous indique les modes de fonctionnement du cryostat.

Les températures ont été repérées au moyen d'une résistance au carbone (Allen Bradley $68 \Omega$ ) logée dans le porte-échantillon. Le tableau montre qu'en fonctionnement classique (hélium liquide en E1, hélium gazeux en E2), en l'absence de toute puissance dissipée au niveau de la cavité, la température minimale atteinte est de 5,4 K. L'écart entre cette température et celle du bain provient du rayonnement de la fenêtre à 300 oK sur la cavité, les autres fenêtres laissant passer la partie du spectre d'émission inférieure à $4 \mu$.

Nous observons en outre, sous irradiation ultraviolette, un échauffement de l'échantillon de 2 oK environ. même après filtrage du visible et de l'infrarouge. Pour travailler à des températures inférie:ıres à $5,4 \mathrm{o}$, nous sommes amenés à introduire de l'hélium dans le tube central E2, la consommation étant alors de $40 \mathrm{l}$ d'hélium gazeux par heure. Dans tous les cas, lors de la mise en froid, il s'avère utile de mettre de l'hélium liquide dans le tube central E2 afin d'atteindre plus rapidement le régime d'équilibre de température du cryostat. En effet, le calcul 


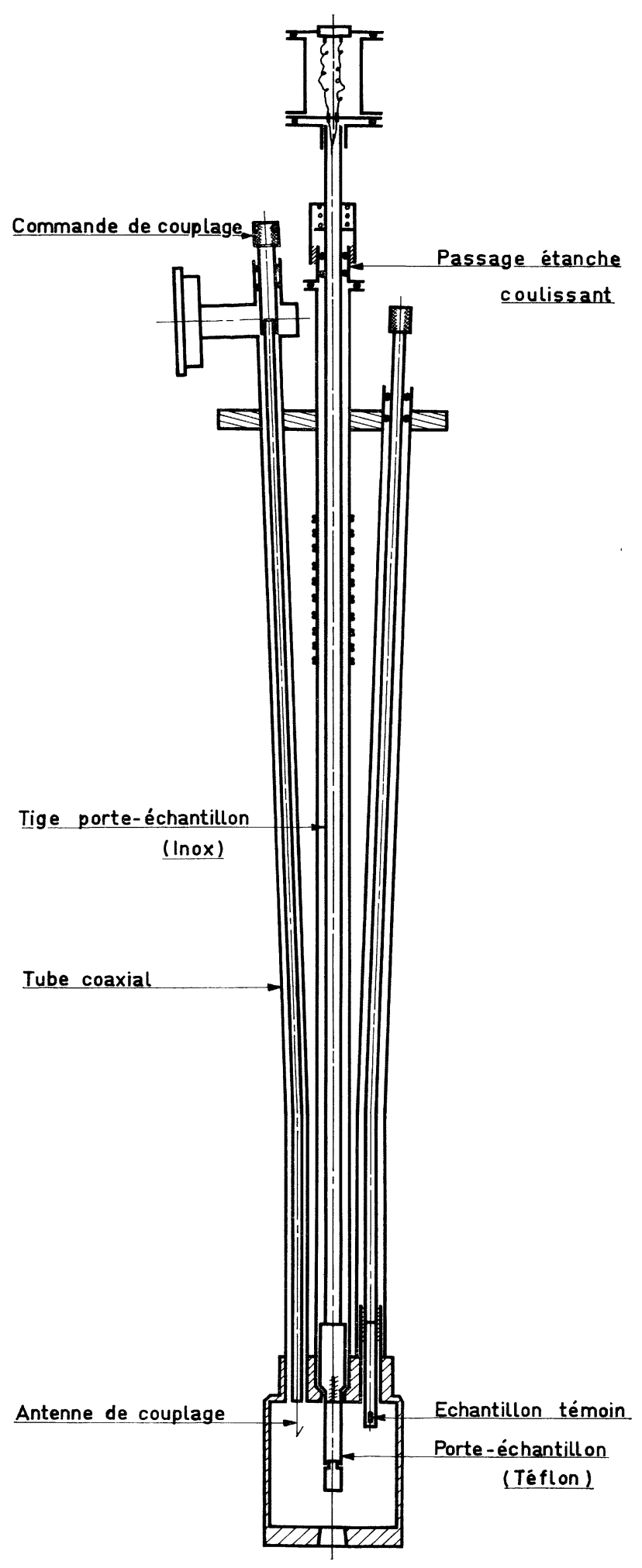

Fig. 1.

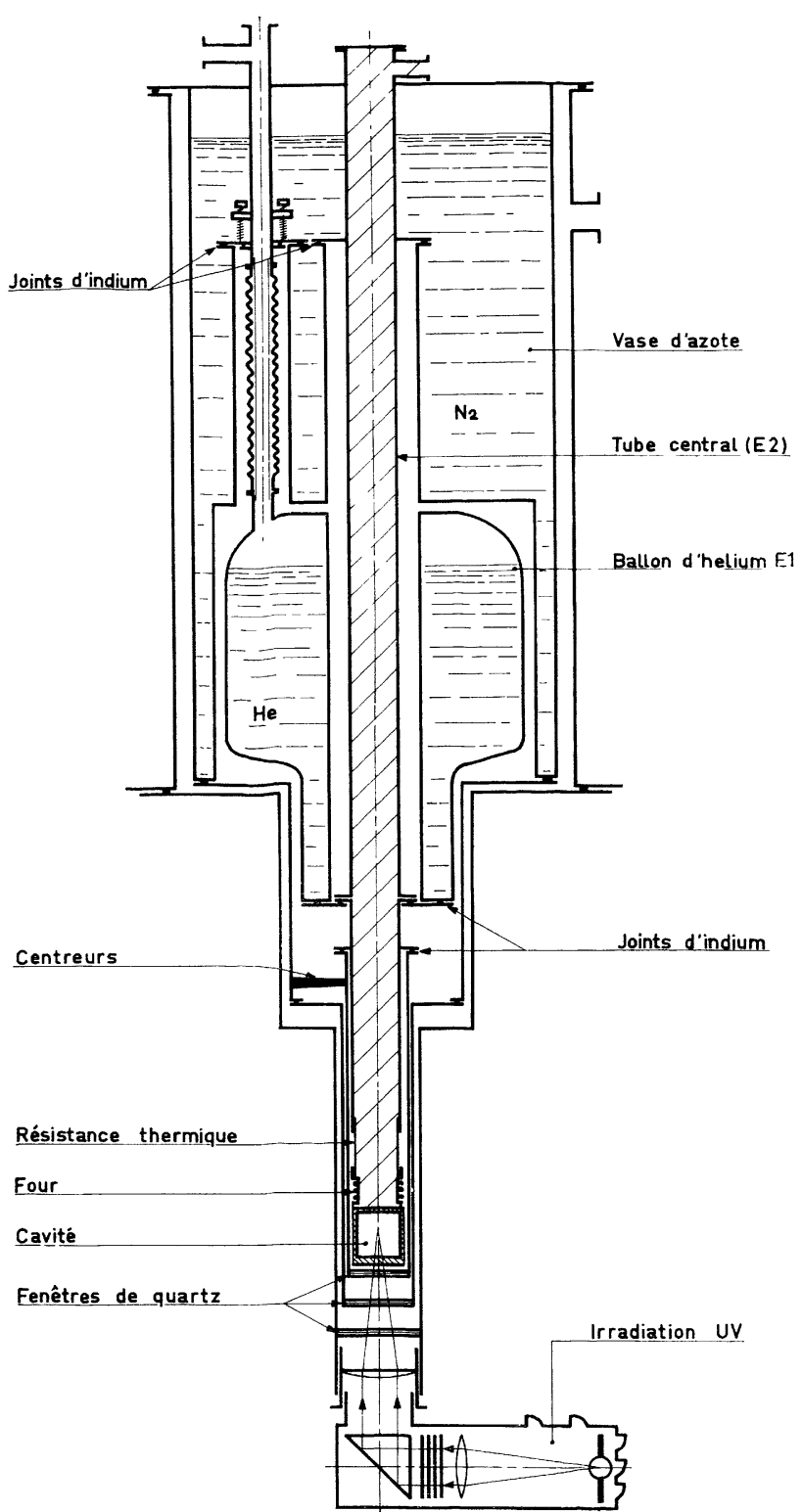

FIG. 2.

montre que l'évolution de la distribution de température le long du tube central, celui-ci étant sous vide, est extrêmement lente, ce que l'expérience confirme.

La variation de la température entre 5,4 et 40 oK (ou 20,6 et $80^{\circ} \mathrm{K}$ ) est obtenue en alimentant le four [situé juste au-dessus de la cavité (fig. 1)], et en évacuant l'hélium gazeux du tube central. En dessous de $20^{\circ} \mathrm{K}$ l'inertie de l'ensemble cavité four - résistance thermique est faible, ce qui permet d'obtenir rapidement une température stable pour un courant donné. Au-dessus de cette température l'inertie devient considérable, et il est nécessaire 
Fluide cryogénique

$$
\text { Température minimale }
$$

Gamme de températures

$\begin{array}{ccc} & 1,3 \text { à } 4,2 \mathrm{o} & <0,01 \mathrm{oK}^{\circ} \\ 4,2 \text { à } 20 \mathrm{oK} & - \\ \text { Fluctuations } & & - \\ \text { de température } & 20 \text { à } 40 \mathrm{o} \mathrm{K} & - \\ & 40 \text { à } 80^{\circ} \mathrm{K} & -\end{array}$

$$
\begin{aligned}
& \text { He liq. en } \mathrm{E} 2 \\
& 1,3{ }^{\circ} \mathrm{K} \\
& 1,3-4,2^{\circ} \mathrm{K}
\end{aligned}
$$$$
\text { Hélium (en E1) }
$$$$
\text { He gaz en E2 }
$$$$
5,4 \text { oK }
$$$$
(p=760 \mathrm{~mm} \mathrm{Hg})
$$$$
5,4-40 \circ \mathrm{K}
$$$$
\left(p<10^{-2} \mathrm{~mm} \mathrm{Hg}\right)
$$$$
<\overline{0,01} \text { oK }
$$$$
\text { (sans régulation) }
$$$$
<0,06 \text { oK }
$$$$
\text { (avec régulation) }
$$

d'employer une régulation de température afin d'atteindre plus vite les températures désirées.

Le transfert d'un échantillon irradié en pile piscine (ou en cellule $\gamma$ ) à la température de l'azote liquide est effectué sans réchauffement. L'échantillon préalablement fixé sous azote liquide à l'ensemble porte-échantillon, est introduit rapidement dans l'enceinte E2. Celle-ci est soumise à une légère pression d'hélium gazeux, qui s'échappe par l'orifice d'introduction. L'échantillon peut être recuit à des températures comprises entre $80^{\circ}$ et $300^{\circ} \mathrm{K}$, en remontant la tige porte-échantillon, grâce à un joint coulissant, jusqu'à un petit four bobiné sur le tube de guidage.

Des tiges porte-échantillon particulières, constituées par un conducteur coaxial, terminées par une
HYDrogène (en E1)

$$
\begin{gathered}
20, \overline{6} \text { oK } \\
20,6-80 \text { oK } \\
\left(p<10^{-2} \mathrm{~mm} \mathrm{Hg}\right) \\
- \\
- \\
<0,2 \text { oK } \\
\text { (avec régulation) }
\end{gathered}
$$

boucle entourant l'échantillon ont été construites pour obtenir une sensibilité de détection optimale, en modulant le champ magnétique à la fréquence de $100 \mathrm{kHz}$. Nous avons obtenu des champs radiofréquences de 10 gauss sans modification appréciable de la consommation.

Remerciements. - Nous remercions vivement M. Pierre Servoz-Gavin pour l'intérêt et le soutien qu'il a apporté à ce travail. Nous devons également exprimer nos remerciements aux Ateliers de Mécanique du C. E. N.-G. pour l'excellente réalisation de cet appareil. Monsieur Tilo Kester remercie l'OTAN et l'EURATOM pour la bourse qui lui a permis de participer à ce travail.

Manuscrit reçu le 20 avril 1966. 